\title{
Calidad de vida, pérdida ponderal y evolución de las comorbilidades. Estudio comparativo de gastrectomía vertical vs. bypass gástrico. Resultados preliminares
}

\author{
Isabel Sierra F. ${ }^{1}$, Cristina Sancho M. ${ }^{1}$, Raúl Juan Cánovas DL. ${ }^{1}$ y Francisco Asencio A. ${ }^{1}$
}

IServicio de Cirugía General y Aparato Digestivo. Hospital de Arnau- Llíria, Valencia,

España.

Recibido 2020-08-02 aceptado 2020-11-09

Correspondencia a: Dra. Isabel Sierra F isa.sierra92@gmail.com
Quality of life, weight loss and evolution of comorbidities. Comparative study of sleeve vs bypass. Preliminary results

Aim: The purpose of our study was to compare the postoperative quality of life, weight loss and improvement of co-morbidities in laparoscopic Roux-en-Y gastric bypass (LRYGB) and Laparoscopic sleeve gastrectomy (LSG) patients one year after surgery. Materials and Method: Match pair analysis of the prospectively collected database of the 23 gastric bypass and 37 gastric sleeve patients operated on in our hospital was performed. Weight loss, quality of life and improvement of co-morbidities were measured at one year after surgery. The quality of life parameters were measured with two standard questionnaires: Gastrointestinal Quality of Life Index (GIQLI) and Bariatric Analysis and Reporting Outcomes System (BAROS). Results: After one year of follow-up the mean BMI was $29.9 \pm 4 \mathrm{~kg} / \mathrm{m}^{2}$ in LRYGB and $31.3 \pm 5 \mathrm{~kg} / \mathrm{m}^{2}$ in LSG. The percent excess BMI Loss (\%EBMIL) was $74.5 \pm 19.2 \%$ (LRYGB) and $67.5 \pm 23.1 \%$ (LSG). A success score in BAROS was obtained in $87 \%$ (LRYGB) and $83.8 \%$ (LSG). The mean GIQLI score was $108.3 \pm 19$ (LRYGB) and $109.8 \pm 18.3$ (LSG). These results did not differ significantly. Remission of co-morbidities was similar in the BGYRL and LSG groups ( 50 vs $53.8 \%$ ). Conclusions: Both types of surgery are effective in quality of life, co-morbidities and weight loss after one year of follow-up. The LRYGB produced better results in \%EBMIL and BAROS, and the LSG produced better results in GIQLI (overall and digestive symptoms). These results did not differ significantly.

Key words: morbid obesity; sleeve gastrectomy; gastric bypass; bariatric surgery; quality of life.

\section{Resumen}

Objetivo: Comparar los resultados obtenidos en calidad de vida, pérdida ponderal y resolución de comorbilidades al año, en pacientes con obesidad mórbida intervenidos de Gastrectomía vertical laparoscópica (GVL) o bypass gástrico en Y de Roux laparoscópico (BGYRL) en nuestro centro. Materiales y Método: Estudio observacional de una base de datos prospectiva. Las variables del estudio fueron IMC pre y posoperatorio, porcentaje de exceso de IMC perdido (PEIMCP), puntuación obtenida en los cuestionarios Gastrointestinal Quality of Life Index (GIQLI) y Bariatric Analysis and Reporting Outcomes System (BAROS) y la resolución de las comorbilidades. Resultados: De 60 pacientes: 37 fueron intervenidos de GVL y 23 de BGYRL. El IMC posoperatorio al año fue $29,9 \pm 4 \mathrm{~kg} / \mathrm{m}^{2}$ en BGYRL y $31,3 \pm 5 \mathrm{~kg} / \mathrm{m}^{2}$ en GVL. El PEIMCP fue de 74,5 $\pm 19,2 \%$ (BGYRL) y de $67,5 \pm 23,1 \%$ (GVL). Con BAROS, se obtuvieron resultados buenos o superiores en el $87 \%$ (BGYRL) y en $83,8 \%$ (GVL). Con GIQLI se obtuvo una puntuación media de 108,3 \pm 19 (BGYRL) y 109,8 $\pm 18,3$ (GVL). No se observaron diferencias estadísticamente significativas entre ambos grupos en ninguna de las variables previas. En cuanto a la evolución de las comorbilidades, $50 \%$ de BGYRL y 53,8\% de GVL presentaron resolución de todas las comorbilidades. Conclusiones: Ambas técnicas son eficaces en cuanto al PEIMCP, a la calidad de vida y al control de comorbilidades al año de la intervención. El BGYRL presenta mejores resultados en PEIMCP y BAROS, y la GVL presenta mejor puntación global y especifica de síntomas digestivos con GIQLI, sin ser diferencias estadísticamente significativas.

Palabras clave: obesidad mórbida; gastrectomía vertical; bypass gástrico; cirugía bariátrica; calidad de vida. 


\section{Introducción}

La cirugía bariátrica es el único tratamiento eficaz en pacientes con obesidad mórbida, que asocian un gran número de comorbilidades, baja autoestima, mala calidad de vida y elevados costes socio sanitarios, en cuanto a la pérdida de peso a largo plazo, reducción de comorbilidades y mejoría de la calidad de vida. Esta mejora en la calidad de vida y percepción de la salud se observa desde el primer año posoperatorio y se mantiene hasta los 10 años de la cirugía. Para el paciente, además de la propia pérdida de peso, el cambio en la calidad de vida es el principal parámetro sobre el cual se centra el éxito subjetivo de la cirugía ${ }^{1}$.

El número de intervenciones en cirugía bariátrica ha aumentado considerablemente en la última década. BGYRL ha sido considerado el patrón oro, pero en los últimos años la GVL ha ganado más popularidad por su sencillez técnica, buenos resultados clínicos y en calidad de vida ${ }^{2}$.

El diferente impacto de ambas técnicas quirúrgicas en los resultados clínicos y en la calidad de vida de los pacientes sigue siendo objeto de discusión. Por ello, en nuestro centro se ha puesto en marcha este estudio con el objetivo de analizar y comparar los resultados obtenidos en calidad de vida, pérdida ponderal y comorbilidades, en pacientes intervenidos de forma primaria con ambas técnicas. Presentamos los resultados recogidos al primer año de la intervención.

\section{Materiales y Método}

\section{Diseño del estudio}

Estudio observacional prospectivo en pacientes con obesidad mórbida intervenidos de cirugía bariátrica de forma consecutiva por el mismo equipo quirúrgico entre enero de 2017 y enero de 2019.

\section{Selección de pacientes}

68 pacientes consecutivos con obesidad mórbida fueron intervenidos de forma primaria con una de las dos técnicas descritas anteriormente (BGYRL o GVL). Ocho pacientes fueron excluidos del estudio porque no completaron los cuestionarios de calidad de vida. De los 60 restantes, 37 se intervinieron con GVL y 23 con BGYRL (Figura 1).

\section{Protocolo del estudio}

Todos los pacientes cumplieron los criterios de inclusión según el Consenso del National Institutes of Health, 199133. La elección de la técnica quirúr- gica se realiza, en función del IMC, comorbilidad y edad según el protocolo del Centro.

Se aplica el protocolo de recuperación intensificada de cirugía bariátrica, dándose de alta al $2 .^{\circ}$ o $3 .^{\text {er }}$ día posoperatorio con dieta líquida especialmente adaptada al posoperatorio. Las revisiones posoperatorias se realizan a los 15 días, al primer mes y cada 3 meses durante el primer año y posteriormente cada año.

El control posoperatorio es multidisciplinar $(\mathrm{Ci}-$ rugía, Endocrinología y Neumología) incluyendo: analítica completa con estudio nutricional, análisis de comorbilidades y cuestionario sobre la calidad de vida anual.

Las complicaciones posoperatorias y su gravedad se evalúan utilizando la clasificación de ClavienDindo ${ }^{4}$.

\section{Técnica quirúrgica}

A todos los pacientes se les realiza la técnica bariátrica estandarizada en el servicio por dos cirujanos de la Unidad de Cirugía Bariátrica.

La GVL se inicia a $4 \mathrm{~cm}$ de píloro, hasta una distancia de sección a $1 \mathrm{~cm}$ del ángulo de His, visualizando el pilar izquierdo, con endograpadora lineal, refuerzo hemostático y tutorizada con sonda intragástrica de $36 \mathrm{Fr}$. No se deja de forma sistemática drenaje intraabdominal ni tampoco sondaje nasogástrico.

En el bypass gástrico en Y de Roux laparoscópico todo el procedimiento se realiza en el espacio supramesocólico (Técnica de Lönroth). Se elabora el reservorio gástrico de unos 45-50 cc y la anastomosis gastroyeyunal con endograpadora lineal. La longitud del asa alimentaria es de $150 \mathrm{~cm}$ y la del

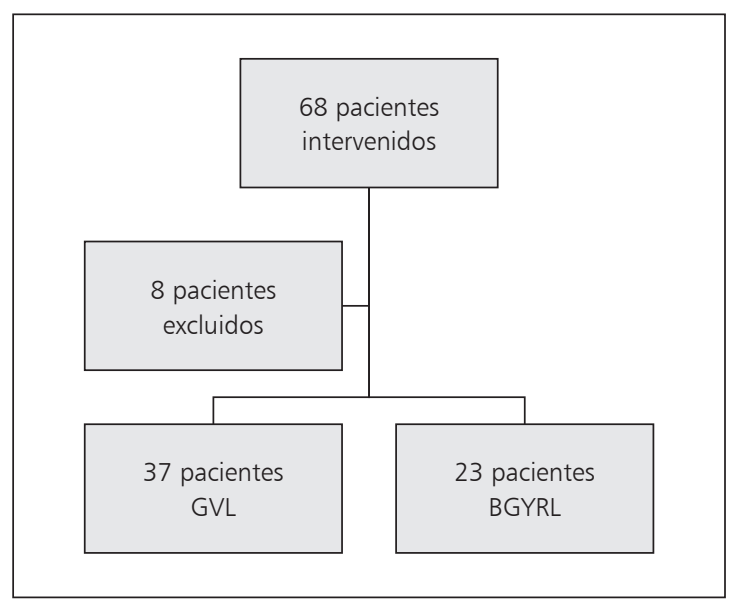

Figura 1. Diagrama de flujo. Selección de pacientes. GVL (gastrectomía vertical laparoscópica) y BGYRL (bypass gástrico en Y de Roux laparoscópico). 
asa biliopancreática de $80 \mathrm{~cm}$. No se realiza el cierre de orificios mesentéricos.

\section{Medidas de resultado}

Las variables principales del estudio son: IMC pre y posoperatorio; el porcentaje de exceso de IMC perdido (PEIMCP) anual; la resolución de las comorbilidades y la puntuación del cuestionario Gastrointestinal Quality of Life Index (GIQLI) y del cuestionario Bariatric Analysis and Reporting Outcomes System (BAROS). Todas las variables posoperatorias se han analizado al año de la intervención.

La pérdida ponderal se ha analizado con el porcentaje del exceso de IMC perdido (PEIMCP), según la fórmula [(IMC inicial - IMC actual/IMC inicial- 25) x 100]; se han clasificado los resultados considerando $>50 \%$ como buenos y $<50 \%$ como fracaso de la cirugía al año de la intervención ${ }^{5}$.

Las comorbilidades estudiadas han sido: hipertensión (HTA), diabetes mellitus tipo 2 (DM2), dislipemia (DL), síndrome de apnea obstructiva del sueño (SAOS) y enfermedad por reflujo gastroesofágico (ERGE). Tras la intervención se han clasificado en: resolución, mejoría, sin cambios o agravadas según los objetivos estándar publicados en 2015 por ASMBS Clinical Issues Committee ${ }^{6}$.

El cuestionario GIQLI evalúa la calidad de vida de forma general y específicamente en el aspecto gastrointestinal. Se compone de 36 preguntas con 5 posibles respuestas cada una. Se obtiene una puntuación global que comprende de 0 (peor resultado) a 144 puntos (mejor resultado) ${ }^{7}$. Engloba 5 áreas: bienestar físico, digestión, hábitos intestinales, bienestar mental y elementos no agrupados. Se ha considerado una puntuación $>100$ como valor de buen resultado ${ }^{8}$.

El cuestionario BAROS se compone de una plantilla visual específica para la obesidad mórbida que evalúa los resultados de la cirugía bariátrica mediante el análisis de tres dominios: la pérdida de peso mediante el porcentaje de peso perdido; la evolución de las comorbilidades; la calidad de vida mediante el Moorehead- Ardelt Quality of Life Ins-

Tabla 1. Análisis de los resultados de pérdida ponderal

\begin{tabular}{|lccc|}
\hline & BGYRL & GVL & p \\
& $\overline{\mathbf{x}} \pm \mathbf{D S}$ & $\overline{\mathrm{x}} \pm \mathbf{D S}$ & \\
\hline IMC Preoperatorio $\left(\mathrm{kg} / \mathrm{m}^{2}\right)$ & $44,7 \pm 5$ & $44,1 \pm 5$ & $\mathrm{p}=0,67$ \\
IMC Posoperatorio $\left(\mathrm{kg} / \mathrm{m}^{2}\right)$ & $30 \pm 4$ & $31,3 \pm 5$ & $\mathrm{p}=0,27$ \\
PEIMCP & $74,5 \pm 19$ & $67,5 \pm 23$ & $\mathrm{p}=0,23$ \\
\hline
\end{tabular}

trument; las complicaciones y las reintervenciones. La puntuación numérica varía entre 0 y 9 puntos. Sus resultados se clasifican en excelente, muy bueno, bueno, regular o fallo según puntuación, siendo una escala diferente dependiendo de la presencia o no de comorbilidades ${ }^{9}$.

\section{Estudio estadístico}

Los valores se han expresado como media \pm desviación estándar.

Se ha utilizado la prueba T Student para comparar las variables cuantitativas y el análisis chicuadrado para las variables cualitativas.

Se ha considerado un valor $\mathrm{p}<0,05$ como significación estadística.

Los datos se han analizado utilizando SPSS Statistics for Macintosh (IBM, versión 22).

\section{Resultados}

De los 60 pacientes estudiados, 37 (61\%) han sido intervenidos de GVL y 23 (39\%) de BGYRL.

El $81,7 \%$ son mujeres y el $18,3 \%$ hombres; la mediana de edad ha sido de 46,5 $\pm 23,64$. Ninguno de los pacientes ha presentado complicaciones mayores posoperatorias (Clavien-Dindo $\geq \mathrm{III}$ ).

El IMC preoperatorio ha sido de $44,7 \pm 5 \mathrm{~kg} / \mathrm{m}^{2}$ en el grupo de BGYRL y de 44,1 $\pm 5,1 \mathrm{~kg} / \mathrm{m}^{2}$ en GVL.

A pesar de los criterios de selección, no se han encontrado diferencias estadísticamente significativas entre los grupos al inicio del estudio en cuanto a IMC preoperatorio, edad y sexo de los pacientes, mientras que son heterogéneas en cuanto a comorbilidades.

\section{Pérdida ponderal}

Hemos constatado un descenso significativo del IMC tras la cirugía en todos los pacientes $(13,5 \pm$ $4,9 \mathrm{~kg} / \mathrm{m}^{2}$ ) (Tabla 1).

Se obtuvieron buenos resultados (PEIMCP $>50 \%$ ) al año de la intervención en 21 pacientes $(91,3 \%)$ de BGYRL y en 31 pacientes $(83,8 \%)$ de GVL, asimismo, se obtuvieron malos resultados (PEIMCP $<50 \%$ ) en 2 pacientes $(8,7 \%)$ de BGYRL y 6 pacientes $(16,2 \%)$ de GVL. Los resultados se consideraron excelentes (PEIMCP $>65 \%$ ) en 16 pacientes $(69,6 \%)$ de BGYRL y en 22 pacientes $(59,5 \%)$ de GVL.

\section{Resolución de las comorbilidades}

Veintidós pacientes $(95,7 \%)$ intervenidos de BGYRL y 26 pacientes $(70,3 \%)$ intervenidos 
Tabla 2. Evolución de comorbilidades según técnica quirúrgica

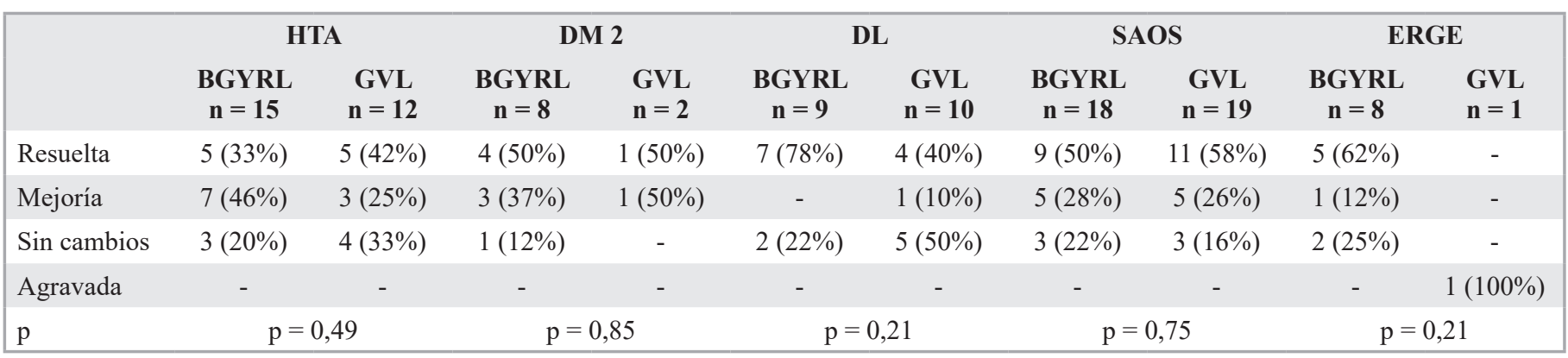

HTA (hipertensión), DM2 (diabetes mellitus tipo 2), SAOS (síndrome de apnea obstructiva del sueño), ERGE (enfermedad por reflujo gastroesofágico).

de GVL presentaron comorbilidades antes de la cirugía. Se ha logrado la resolución de todas las comorbilidades en 11 pacientes $(50 \%)$ de BGYRL y 14 pacientes $(53,8 \%)$ de GVL; mejoría en 10 pacientes $(45,5 \%)$ de BGYRL y 11 pacientes $(42,3 \%)$ de GVL.

La Tabla 2, muestra los resultados obtenidos desglosados por comorbilidades.

Independientemente de la técnica quirúrgica realizada, los pacientes con un PEIMCP $\geq 50 \%$ (41 pacientes) han presentado resolución de comorbilidades 24 de ellos $(58,5 \%)$ y, mejoría, 17 pacientes $(41,5 \%)$, resultado significativamente superior frente a los que han obtenido un PEIMCP $<50 \%$.

En nuestro estudio, 9 pacientes presentaron ERGE sintomático preoperatorio. A 8 se les realizó BGYRL y a 1 GVL. De los 8 pacientes intervenidos de BGYRL, 6 (75\%) presentaron resolución/mejoría de los síntomas de ERGE, 5 de estos pacientes obtuvieron una buena puntuación BAROS.

\section{Puntuación en cuestionarios de calidad de vida}

En la Tabla 3 se muestran los resultados obtenidos en ambos cuestionarios.

Los pacientes con mayor PEIMCP $(>65 \%)$ al año, ha obtenido mejor puntuación en BAROS, de forma significativa $(\mathrm{p}<0,01)$.

\section{Discusión}

En nuestro centro, las técnicas quirúrgicas utilizadas para la cirugía bariátrica son el BGYRL (39\%) y la GVL (61\%); ambas técnicas son las más populares a nivel mundial como muestra el último registro publicado de la IFSO: la más utilizada es la gastrectomía vertical $(53,6 \%)$ seguida del bypass gástrico $(30,1 \%)^{10}$.

A pesar de que la obesidad es más común en
Tabla 3. Análisis de los resultados en cuestionarios de calidad de vida

\begin{tabular}{|lccc|}
\hline & $\begin{array}{c}\text { BGYRL } \\
\overline{\mathbf{x}} \pm \mathbf{D S}\end{array}$ & $\begin{array}{c}\text { GVL } \\
\overline{\mathbf{x}} \pm \mathbf{D S}\end{array}$ & $\mathbf{p}$ \\
\hline Puntuación total BAROS & $4,6 \pm 1,7$ & $4,5 \pm 1,6$ & $\mathrm{p}=0,77$ \\
Puntuación total GIQLI & $108,3 \pm 19$ & $109,8 \pm 18$ & $\mathrm{p}=0,77$ \\
Síntomas digestivos GIQLI & $54,3 \pm 9$ & $56 \pm 9$ & $\mathrm{p}=0,49$ \\
& $\mathbf{N}(\%)$ & $\mathbf{N}(\%)$ & \\
BAROS (bueno o superior) & $20(87 \%)$ & $31(84 \%)$ & $\mathrm{p}=0,5$ \\
GIQLI $>100$ & $16(70 \%)$ & $27(73 \%)$ & $\mathrm{p}=0,77$ \\
\hline
\end{tabular}

hombres que en mujeres ${ }^{11}$, es mayor el porcentaje de mujeres con obesidad mórbida que se intervienen. En nuestro estudio el $82 \%$ han sido mujeres y el $18 \%$ hombres.

Para la valoración de la pérdida ponderal, el IMC tiene bastantes limitaciones, ya que deja al margen la composición corporal de cada individuo. En 2004, se propuso el concepto «porcentaje de exceso del IMC perdido» (PEIMCP) $)^{5}$, medida que hemos utilizado en nuestro estudio.

Independientemente del procedimiento, hemos obtenido un descenso significativo de $13,5 \mathrm{~kg} / \mathrm{m}^{2}$ en el IMC posoperatorio al año. Estos datos concuerdan con la literatura revisada ${ }^{12}$, al igual que el PEIMCP al año; los estudios publicados con revisión a corto plazo muestran un PEIMCP al año del 76,6 $\pm 21 \%$ en el BGYRL y del $68 \pm 29 \%$ en GVL ${ }^{8,13}$.

Aunque no hay una estandarización para la medición de la pérdida ponderal; los resultados que hemos obtenido con PEIMCP al año cumplen con los estándares de calidad y con los objetivos según las recomendaciones de consenso de la Sociedad Española de Cirugía de la Obesidad (SECO); pueden considerarse excelentes con PEIMCP $>65 \%$ en 
el $70 \%$ (BGYRL) y $60 \%$ (GVL) de los pacientes intervenidos al año ${ }^{5}$.

Peterli en 2017, presentó un estudio prospectivo comparando la GVL vs. BGYRL. Los resultados reflejaron un PEIMCP del $72,3 \pm 22 \%$ vs. $76,6 \pm 21 \%$ $(\mathrm{p}=0,139)$ considerando el nadir entre 1-2 años y experimentando reganancia en ambas técnicas a los 3 años de seguimiento, sin encontrar diferencias significativas entre ambos grupos ${ }^{8}$.

En la literatura, no hay claramente superioridad de una técnica respecto a la otra en cuanto a resolución de las comorbilidades, y como ocurre en nuestro estudio, las comorbilidades responden más a la pérdida de peso que a la técnica quirúrgica.

Li ha publicado un metaanálisis analizando los resultados a largo plazo de GVL vs. BGYRL de 32 estudios (6.526 pacientes), los resultados son similares en resolución de DM y SAOS, muestra superioridad del BGYRL en el control de HTA, DL, ERGE y artritis, pero a expensas de mayor número de complicaciones y de reintervenciones. En cuanto al análisis de la ERGE recomiendan realizar BGYRL basados en el porcentaje de resolución (78,2\% BGYRL vs. $28 \%$ GVL) ${ }^{14}$.

En los estudios a corto plazo, el Swiss Multicenter Bypass or Sleeve Study (SM -BOSS) muestra porcentajes de resolución de comorbilidades mayores similares para ambas técnicas a los 3 años salvo la ERGE y la DL, donde los resultados son mejores para el BGYR ${ }^{8}$.

Nuestros resultados son similares, el 50\% (BGYRL) y el 54\% (GVL) presentaron resolución completa de todas las comorbilidades y el $45 \%$ (BGYRL) y 42\% (GVL) presentaron mejoría, sin diferencias significativas entre ambos grupos. Además, los pacientes con DM e HTA presentaron resolución o mejoría en un porcentaje superior a los objetivos recomendados 5 .

Cuando desglosamos nuestros resultados (Tabla 2) constatamos que ambas técnicas son eficaces en resolución/mejoría de las comorbilidades analizadas, y aunque sin ser significativo, observamos mejor control de la DL en el BGYRL.

La mayoría de los grupos recomiendan el BGYRL en pacientes con ERGE porque uno de los inconvenientes de la GVL es el potencial desarrollo o empeoramiento de ERGE posoperatorio ${ }^{15,16}$.

Biter concluye que la GVL presenta más sintomatología asociada al reflujo gastroesofágico, pero esto no influye en la calidad de vida ${ }^{17}$.

Con nuestros datos, siendo la muestra tan pequeña, no podemos afirmar una clara relación entre la calidad de vida de los pacientes y la evolución de la ERGE.
Independientemente del procedimiento realizado, observamos una relación directa y significativa entre un mayor PEIMCP al año, la resolución y/o mejoría de todas las comorbilidades y una mejor puntuación en el cuestionario de calidad de vida BAROS.

La calidad de vida mejora desde el primer año del posoperatorio y se mantiene incluso hasta los 10 años de la cirugía, siendo uno de los factores que más influye en la valoración subjetiva del paciente ${ }^{2}$.

Para evaluar la calidad de vida existen varios instrumentos, algunos más específicos que otros y con mucha heterogeneidad entre ellos ${ }^{18}$.

Rausa publica una revisión sistemática sobre calidad de vida tras GVL y BGYRL con un seguimiento a medio plazo (11 estudios con un total de 3.145 pacientes) y concluyen que los más utilizados son el GIQLI y el SF-36. Independientemente de la técnica hay un aumento de la puntuación en el posoperatorio (18 meses), sin embargo, la trayectoria observada decrece con el paso del tiempo (durante los 4 años posteriores a la cirugía) ${ }^{2}$. Al analizar los síntomas específicos se observa mejor puntuación en la GVL vs BGYRL en los síntomas digestivos (65 vs. 58), hábitos digestivos (27 vs. $23)$ y en actividad física (22,5 vs. 20) a los 3 años de la intervención.

Tayyem, hace una revisión de los cuestionarios más utilizados. Hemos decidido utilizar el GIQLI y el Moorhead-Ardelt incluido en el cuestionario BAROS según recomendaciones de la $\mathrm{SECO}^{19}$.

En nuestros resultados, no hemos obtenido diferencias significativas entre ambas técnicas y al igual que en la literatura hemos obtenido mejor puntuación en la GVL vs. BGYRL en los síntomas digestivos (56 vs. 54).

Un punto fuerte de este estudio es el análisis de una base de datos prospectiva, cuyos pacientes han sido intervenidos únicamente por dos cirujanos experimentados en ambas técnicas, lo que limita la variabilidad de la técnica quirúrgica, asimismo pocos estudios en la literatura actual comparan de forma prospectiva los resultados en calidad de vida entre BGYRL y GVL 3 .

Una limitación importante del estudio es la imposibilidad de aleatorización debido a la diferente elección de la técnica quirúrgica según el IMC y comorbilidades, siguiendo nuestro protocolo de actuación. Otras limitaciones del estudio han sido el pequeño tamaño muestral y la falta de registro de calidad de vida preoperatoria.

La valoración de la calidad de vida en personas intervenidas de cirugía bariátrica es compleja y subjetiva, se deben crear cuestionarios y escalas propias para los pacientes con obesidad mórbida que definan 
mejor las expectativas y problemas específicos de la cirugía bariátrica.

\section{Conclusión}

Ambas técnicas son eficaces en cuanto al PEIMCP, a la calidad de vida y al control de comorbilidades al año de la intervención. El BGYRL presenta mejores resultados en PEIMCP y BAROS, y la GVL presenta mejor puntación global y especifica de síntomas digestivos con GIQLI, sin ser diferencias estadísticamente significativas.

\section{Responsabilidades éticas}

Protección de personas y animales. Los autores declaran que para esta investigación no se han realizado experimentos en seres humanos ni en animales.

Confidencialidad de los datos. Los autores declaran que en este artículo no aparecen datos de pacientes.

Conflictos de interés: no hay.

\section{Bibliografía}

1. Domínguez-Adame Lanuza E. Estándares de calidad en la morbilidad, mortalidad y calidad de vida. Máster en Cirugía de la Obesidad 2019-2020. Editorial Médica Panamericana.

2. Rausa E, Kelly ME, Galfrascoli E, Aiolfi A, Cavalcoli F, Turati L, et al. Quality of Life and Gastrointestinal Symptoms Following Laparoscopic Roux-en-Y Gastric Bypass and Laparoscopic Sleeve Gastrectomy: a Systematic Review. Obes Surg. 2019;29:1397-402. doi: 10.1007/ s11695-019-03737-3.

3. Am J Clin. Gastrointestinal surgery for severe obesity: National Institute of Health Consensus Development Conference Statement. Nutr 1992; 55 (suppl 2): 615S-9S. doi: 10.1093/ ajen/55.2.615s.

4. Dindo D, Demartines N, Clavien PA. Classification of surgical complications: a new proposal with evaluation in a cohort of 6336 patients and results of a survey.Ann Surg. 2004;240:205-13. doi: 10.1097/01. sla.0000133083.54934.ae.

5. Sabench Pereferrer F, Domínguez-Adame Lanuza E, Ibarzabal A, Socas Macias M, Valentí Azcárate V, García Ruiz de Gordejuela A, et al. Criterios de calidad en cirugía bariátrica: revisión de conjunto y recomendaciones de la Asociación Española de Cirujanos y de la Sociedad Española de Cirugía de la Obesidad. Cir Esp. 2017;95:4-16. doi: 10.1016/j. ciresp.2016.09.007.

6. for the ASMBS Clinical Issues Committee, Brethauer SA, Kim J, el Chaar M, Papasavas P, Eisenberg D, et al. Standardized Outcomes Reporting in Metabolic and Bariatric Surgery. Obes Surg. 2015;25:587-606. doi: 10.1007/ s11695-015-1645-3.

7. Biter LU, van Buuren MMA. Quality of life 1 year after laparoscopic sleeve gastrectomy versus laparoscopic Roux-en-Y gastric bypass: a randomized controlled trial focusing on gastroesophageal reflux disease. Obes Surg. 2017;27:2557- 65. doi: 10.1007/ s11695-017-2688-4.

8. Peterli R, Wolnerhanssen BK, Vetter D. Laparoscopic sleeve gastrectomy versus Roux-Y-gastric bypass for morbid obesity-3- year outcomes of the prospective randomized Swiss Multicenter Bypass Or Sleeve Study (SM-BOSS). Ann Surg. 2017;265:466-73. doi: 10.1097/ SLA.0000000000001929.

9. Oria HE, Moorehead MK. Bariatric Analysis and Reporting Outcome System (BAROS). Obes Surg. 1998;8:487-99. doi: 10.1381/096089298765554043.

10. Angrisani L, Santonicola A, Iovino P, Vitiello A, Higa K, Himpens J, et al. IFSO Worldwide Survey 2016: Primary, Endoluminal, and Revisional Procedures. Obes Surg. 2018;28(:3783-94. doi: 10.1007/s11695-018-3450-2.

11. Aranceta-Bartrina J, Pérez-Rodrigo C, Alberdi-Aresti G, Ramos-Carrera N, Lázaro-Masedo S. Prevalencia de obesidad general y obesidad abdominal en la población adulta española (25-64 años) 2014-2015: estudio ENPE. Rev Esp Cardiol. 2016;69:579-87. doi: 10.1016/j. rec.2016.02.009.
12. Himpens J, Dapri G, Cadière G. A Prospective Randomized Study Between Laparoscopic Gastric Banding and Laparoscopic Isolated Sleeve Gastrectomy: Results after 1 and 3 Years. Obes Surg. 2006;16:1450-6. doi: 10.1381/096089206778869933.

13. Sánchez-Santos R, Masdevall C, Baltasar A, Martínez-Blázquez C, García Ruiz de Gordejuela A, Ponsi E, et al. Shortand Mid-term Outcomes of Sleeve Gastrectomy for Morbid Obesity: The Experience of the Spanish National Registry. Obes Surg. 2009;19:1203-10. doi: 10.1007/s11695-009-9892-9.

14. Li J, Lai D, Wu D. Laparoscopic Rouxen-Y Gastric Bypass Versus Laparoscopic Sleeve Gastrectomy to Treat Morbid Obesity-Related Comorbidities: a Systematic Review and Metaanalysis. Obes Surg. 2016;26:429-442. doi: 10.1007/s11695-015-1996-9.

15. Pallati PK, Shaligram A, Shostrom VK, Oleynikov D, McBride CL, Goede MR. Improvement in gastroesophageal reflux disease symptoms after various bariatric procedures: Review of the Bariatric Outcomes Longitudinal Database. Surg Obes Relat Dis. 2014;10:502-7. doi: 10.1016/j.soard.2013.07.018.

16. DuPree CE, Blair K, Steele SR, Martin MJ. Laparoscopic Sleeve Gastrectomy in Patients With Preexisting Gastroesophageal Reflux Disease: A National Analysis. JAMA Surg. 2014;149:328. doi: 10.1001/ jamasurg.2013.4323.

17. Biter LU, van Buuren MMA. Quality of life 1 year after laparoscopic sleeve gastrectomy versus laparoscopic 
CALIDAD DE VIDA, PÉRDIDA PONDERAL Y EVOLUCIÓN DE LAS COMORBILIDADES. ESTUDIO COMPARATIVO DE GASTRECTOMÍA ... - I. Sierra F. et al.

\section{ARTÍCULO ORIGINAL}

Roux-en-Y gastric bypass: a

randomized controlled trial focusing on

gastroesophageal reflux disease. Obes

Surg. 2017;27:2557- 65. doi: 10.1007/

s11695-017-2688-4.

18. Tayyem R, Ali A, Atkinson J, Martin
CR. Analysis of Health-Related Qualityof-Life Instruments Measuring the Impact of Bariatric Surgery: Systematic Review of the Instruments Used and their Content Validity. Patient 2011;4:73-87. doi: 10.2165/11584660-000000000-
00000.

19. Sabench F, Molina A, Del Castillo D. Estándares de calidad en la pérdida de peso y resolución de comorbilidades. Máster en Cirugía de la Obesidad 20192020. Editorial Médica Panamericana. 